\title{
Article
}

\section{L'érosion sur la frange côtière : un exemple de gestion des risques}

\author{
Catherine Meur-Férec ${ }^{\mathrm{a}}$, Valérie Morel $^{\mathrm{b}}$ \\ a Géographe, Institut des mers du Nord, EA 1702, Université du Littoral Côte d'Opale, 21 quai de la Citadelle, BP 5528, \\ 59383 Dunkerque cedex 1, France \\ b Géographe, Dynamique des réseaux et des territoires, EA 2468, Université d'Artois, 9 rue du Temple, BP 665, \\ 62030 Arras cedex, France
}

\begin{abstract}
La gestion des risques est une question abordée à plusieurs reprises par NSS. Il s'agit ici de risques non pas technologiques mais naturels, générés par les mouvements contradictoires de la mer et de l'occupation humaine des rivages. Dans l'enchevêtrement des intérêts qui se confrontent sur une frange côtière, on voit surgir une question de fond pour nos sociétés : comment conjuguer protection et prévention avec l'intérêt général dont se réclament tout autant l'élu local, l'État, etc. ? Pour NSS, cette question est d'autant plus vive que les éventuels impacts du changement climatique sur le niveau de la mer lui donneront une acuité encore plus grande. Cet article amorce une telle réflexion.
\end{abstract}

La Rédaction

\section{Mots-clés :}

risques côtiers; érosion: tourisme balnéaire ; Pas-de-Calais ; France

\section{Keywords:}

coastal risks; erosion; seaside tourism; Pas-de-Calais; France
Résumé - Cet article présente une réflexion sur les processus d'émergence et de gestion des risques d'érosion sur la frange côtière, illustrée par l'exemple de la station balnéaire de Wimereux dans le Pas-deCalais. Alors que la dynamique naturelle confère aux côtes une mobilité intrinsèque et, dans la plupart des cas, une tendance au recul vers les terres, l'évolution des installations humaines obéit à une logique inverse de progression vers la mer et de fixité du trait de côte. La conjugaison de forts aléas "naturels » et d'enjeux considérables concentrés sur un espace restreint crée une grande vulnérabilité sur la frange côtière. Aujourd'hui fortement investi, cet espace devient un territoire à risques. L'exemple de Wimereux est révélateur de cette situation résultant du «télescopage» entre processus naturels et anthropiques. Cette dynamique convergente, à l'origine des risques côtiers, reflète une tendance générale observée sur la plupart des littoraux urbanisés. En réponse à cet état de fait, la gestion des risques d'érosion côtière s'est progressivement développée en France, mais elle se heurte à de nombreuses difficultés d'application.

\begin{abstract}
Erosion of the coastline: an example of risk management. This paper presents a reflexion on the newly emerging awareness of erosion risk and its management, as illustrated by the seaside resort of Wimereux, in the north of France. Bearing in mind that nature imposes naturally on beaches an intrinsic mobility, and in most cases, a tendency to inland retreat, the trend of man-made setting-ups on the other hand, imposes the very opposite movement towards the shoreline which becomes then artificially fixed. The combination of unpredictable natural phenomena together with a concentration of shoreline manmade infrastructures creates a coastal area of high risk. Wimereux provides us with a probing example of the "telescoping" of natural and man-made phenomena. This convergent dynamic system, at the origin of risks for the coastlines, reflects a tendency observed on most urbanized coasts. As a response to this hazard, coastline management used in France have made progress but considerable difficulties remain in ensuring their successful application.
\end{abstract}

Les espaces proches du trait de côte (Encadré 1) sont caractérisés par leur mobilité permanente et particulièrement rapide à l'échelle du temps géologique, mais

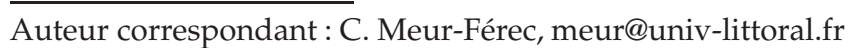

aussi à l'échelle humaine (Pinot, 1998; Paskoff, 1998). Longtemps considérées comme répulsives et qualifiées de «territoire du vide» (Corbin, 1988), les côtes françaises ont suscité un attrait croissant à partir du dixhuitième siècle. Sur ce milieu dynamique, se sont alors 


\begin{abstract}
Encadré 1. La frange côtière
La problématique concerne ici, non pas le « littoral » au sens large (Corlay, 1995 ; Dauvin, 2002 ; Miossec, 1998b), mais spécifiquement la «frange côtière ». Il s'agit d'une bande étroite située à proximité immédiate du trait de côte et directement concernée par sa mobilité. Selon les auteurs et les problématiques, la largeur de cette bande varie. Dans l'étude cartographique réalisée pour le Conservatoire de l'espace littoral et des rivages lacustres (CELRL) (Robin et Verger, 1996), une largeur de $500 \mathrm{~m}$ a été retenue dans le but d'estimer l'évolution de l'urbanisation côtière par analyse diachronique de photographies aériennes. Dans le travail entrepris sur « la vulnérabilité des territoires côtiers » dans le cadre du PNEC, nous avons choisi une largeur de $400 \mathrm{~m}$ pour évaluer les enjeux économiques côtiers (Meur-Férec, 2003a). Cette valeur de $400 \mathrm{~m}$ a été retenue compte tenu des vitesses maximales de recul du trait de côte en France, soit près de 4 mètres par an depuis une cinquantaine d'années, comme en baie de Wissant par exemple (Marquet, 2000 ; Paxion et Cohen, 2002). En conséquence, cette bande de 400 m correspondrait théoriquement à l'espace maximum perdu en un siècle sur les côtes qui reculent le plus rapidement. Ce chiffre est très probablement surestimé et varie sensiblement selon la morphologie de la côte, mais, en l'absence de certitude sur les rythmes d'évolution, il constitue un repère de largeur maximale nécessaire à l'évaluation des enjeux. L'échéance d'un siècle est l'échelle de temps retenue dans le cadre des Plans de prévention des risques (PPR) (MATE, 1997a). Dans tous les cas, ces précisions de largeur, et donc de délimitation de l'espace concerné, sont essentielles car elles conditionnent la signification des données.
\end{abstract}

\title{
Encadré 2. Aléas, enjeux, vulnérabilité, risques
}

Ces termes relèvent de la terminologie de l'étude des risques, mais leurs sens diffèrent parfois sensiblement d'une source à l'autre et évoluent au fil des travaux de recherche. Notre approche se fonde sur les définitions qui émanent du ministère de l'Aménagement du territoire et de l'Équipement (MATE, 1997b) ainsi que sur les travaux de R. D’Ercole et P. Pigeon.

Ainsi, l'aléa est un "événement d'origine naturelle ou humaine potentiellement dangereux dont on essaie d'estimer l'intensité et la probabilité d'occurrence par l'étude des périodes de retour ou des prédispositions du site ». Les enjeux représentent quant à eux la «valeur humaine, économique ou environnementale des éléments exposés à l'aléa » (MATE, op. cit.).

La définition de la vulnérabilité est plus complexe. Dans son acception la plus simple (MATE, op. cit.), c'est la «capacité ou propension à favoriser l'endommagement (pour les biens et les activités) ou les préjudices (pour les personnes) des éléments exposés à l'aléa ». À partir de travaux réalisés dans plusieurs pays en développement, R. D’Ercole et P. Pigeon (1999) proposent une approche plus large qui considère la vulnérabilité des sociétés à travers leur capacité de réponse à des crises potentielles. «Elle traduit la fragilité d'un système dans son ensemble, et de manière indirecte sa capacité à surmonter la crise provoquée par un aléa. » Ainsi, plus un système est apte à se rétablir après une catastrophe et donc à faire preuve de "résilience », moins il est vulnérable.

Le risque résulte de la combinaison d'un aléa avec les enjeux (MATE, op. cit.). En complément de cette définition, les approches de R. D’Ercole et P. Pigeon insistent sur l'importance de la vulnérabilité des sociétés. Ainsi, d'après R. D’Ercole (1994), le risque dépend de facteurs structurels (contexte socio-économique, culturel, fonctionnel ou institutionnel) et conjoncturels (l'aléa) qui influencent la capacité de réponse de la société à l'événement et en font ainsi varier les effets. La dernière définition proposée par P. Pigeon (2002) intègre dans la vulnérabilité les effets sur le terrain des politiques de gestion des risques (prévention et secours). Le risque serait alors « la probabilité d'occurrence de dommage compte tenu des interactions entre processus physiques d'endommagement (aléas) et facteurs de peuplement (vulnérabilité)».

progressivement installées des sociétés occupant un espace étroit et mouvant. Aujourd'hui fortement investie, la frange côtière est devenue un territoire à risques. Les aléas liés à sa mobilité intrinsèque et les enjeux qui s'y concentrent lui confèrent une grande vulnérabilité, caractéristique de nombreux littoraux urbanisés (Encadré 2).

La côte de Wimereux, qui a connu un développement touristique important et ancien (Lageiste, 2002), reflète cette vulnérabilité. Au cours du dix-neuvième siècle, les hommes ont progressivement occupé ce site côtier pour des raisons militaires, puis pour des motivations touristiques. Les installations se sont principalement concentrées au centre de la baie, sur un massif dunaire, entre la pointe de la Rochette au nord et la falaise de la Crèche au sud (Fig. 1). Le site originel des premières implantations humaines sur cette côte est l'estuaire du Wimereux, petit fleuve côtier traversant la partie septentrionale des dunes. Située à $4 \mathrm{~km}$ au nord de Boulogne-sur-Mer, Wimereux compte aujourd'hui 8000 habitants. Le problème de la gestion des risques d'érosion se pose pour cette station balnéaire, comme pour de nombreux sites côtiers urbanisés ${ }^{1}$.

\footnotetext{
${ }^{1}$ Ce texte s'appuie sur une communication présentée lors du colloque du Groupe français de géomorphologie «Dynamiques littorales quaternaires ", qui s'est tenu à Rennes les 27 et 28 mars 2003. Le travail qu'il présente a été financé et réalisé dans le cadre du Programme national d'environnement côtier (PNEC), axe de recherche thématique 6, "Gouvernance environnementale et patrimoine côtier». Cette recherche constitue une approche introductive au programme pluridisciplinaire «La vulnérabilité des territoires côtiers : évaluation, enjeux et politiques publiques» (contrat Ifremer 2002 / 1140 717).
} 


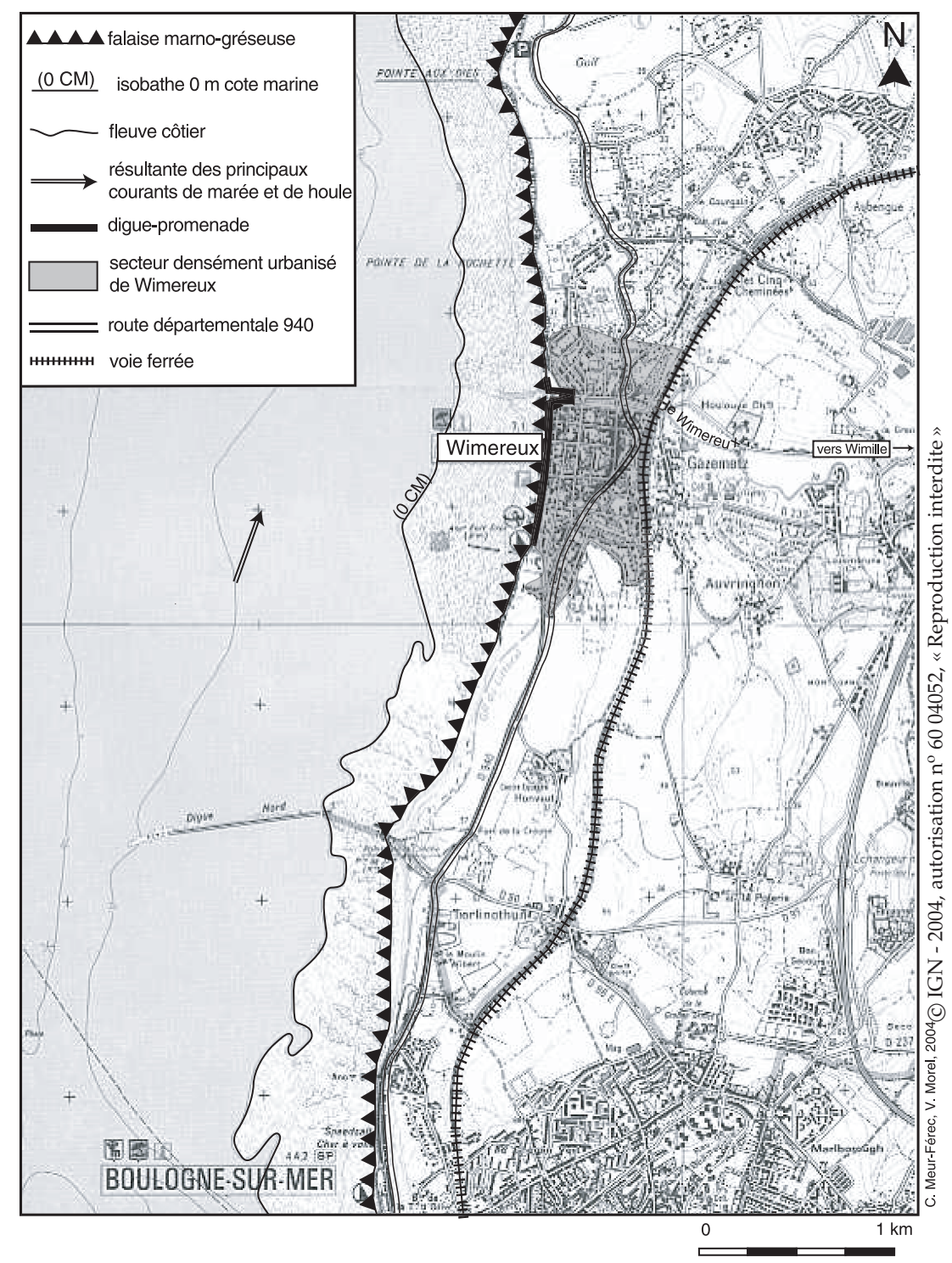

Fig. 1. Présentation simplifiée de la côte de Wimereux (62). Extrait de la carte IGN TOP25 : 2104 ET Boulogne-sur-Mer, 2000.

\section{Aléa érosion et enjeux : des dynamiques convergentes}

\section{Une tendance au recul du trait de côte}

Depuis que l'on mesure de façon fiable l'évolution du rivage, c'est-à-dire au moins un demi-siècle, on constate que de nombreux littoraux marquent une tendance au recul vers la terre. Bien qu'il existe des côtes stables à moyen terme ou même en " progradation » (avancée de la ligne de rivage sur la mer) (Battiau-Queney et al., 2003), cette tendance érosive est généralement admise. Ainsi, d'après Bird (1987), près de $70 \%$ des littoraux sableux du monde seraient en érosion. À ce chiffre, il faut ajouter toutes les côtes à falaise qui, par définition, sont des formes d'érosion et qui ne peuvent évoluer que par recul. Les causes de cette tendance évolutive sont avant tout liées à la dynamique naturelle des côtes (Paskoff, 1993). Les principaux facteurs explicatifs seraient la conjugaison d'une pénurie de sédiments depuis la fin de la transgression postglaciaire, de conditions hydrométéorologiques variables (tempêtes, précipitations) et d'une légère remontée relative du niveau marin (Paskoff, 2001; Pirazzoli, 1993). Cependant, des facteurs anthropiques contribuent couramment à accentuer ce phénomène, notamment l'interruption des échanges dune-estran par l'artificialisation du trait de côte, les effets induits de certains ouvrages de défense contre la mer, les extractions de sédiments marins, les changements d'occupation des sols dans les bassins versants (Sabatier et Suanez, 2003), etc.

À partir de la Seconde Guerre mondiale et de l'utilisation des photographies aériennes, et surtout depuis l'emploi généralisé des logiciels de rectification et de superposition de ces photographies, les variations de la ligne de rivage sont étudiées de plus en plus finement 
(Crowell et al., 1997; Gaillot et Chaverot, 2001; Moore, 2000 ; Robin, 2002). En France et sur les rivages sableux, des reculs moyens situés entre 0,5 et 1 mètre par an sont les plus fréquents, mais ces valeurs peuvent être bien plus fortes ponctuellement, comme au centre de la baie de Wissant (Pas-de-Calais). En ce qui concerne les falaises à recul rapide, les moyennes sur un siècle sont plus significatives en raison du caractère plus brutal des événements (glissement, éboulement). Les chiffres sont là encore très variables selon les contextes lithologiques et dynamiques. Des valeurs situées entre 10 et $30 \mathrm{~m}$ par siècle sont les plus courantes pour les falaises crayeuses (Costa et al., 2002; Hénaff et al., 2002 ; Lahousse et Pierre, 2002; Morel, 2002); mais il n'est pas rare de trouver des vitesses de recul ponctuel bien supérieures, comme à Criel-surMer (Seine-Maritime) où le phénomène $a$ fait s'effondrer jusqu'à 10 m de falaise entre 1995 et 1998 (service maritime de la Direction départementale de l'Équipement, communication personnelle).

Sur la côte de Wimereux, l'analyse et la confrontation de plusieurs documents permettent d'approcher quantitativement et spatialement la tendance au recul depuis près de 150 ans. Les archives du Service maritime des ports de Boulogne et Calais (SMBC), notamment, témoignent de l'ancienneté de cette dynamique érosive. Ainsi, le rapport d'un ingénieur des travaux publics fait état de la situation critique soulevée dès 1884 par les propriétaires riverains : «[...] vingt-huit propriétaires du village de Wimereux exposent à Monsieur le Ministre des travaux publics que la mer menace de plus en plus les propriétés riveraines de la plage de Wimereux et qu'elle a envahi une largeur de $30 \mathrm{~m}$ de terrain depuis la vente de terrain, faite par l'État en 1865 ». Les dunes au centre de la baie reculaient donc déjà au rythme soutenu de 1,5 mètre par an à la fin du dix-neuvième siècle. Cette vitesse n'est aujourd'hui plus mesurable car tout le secteur est artificiellement fixé par une large digue-promenade. Par contre, au pied de la digue, l'épaisseur du haut de plage s'amenuise progressivement, fragilisant l'ouvrage et entraînant la submersion totale de la plage à marée haute. L'érosion n'affecte pas uniquement les dunes du centre de la baie ; les falaises qui l'encadrent, particulièrement au nord de l'estuaire du Wimereux, subissent également un recul marqué, comme en témoigne l'analyse diachronique effectuée par le Centre d'études techniques de l'Équipement (CETE) dans le cadre du projet de Plan de prévention des risques (PPR) (Fig. 2). Les vitesses de recul obtenues atteignent des maxima très élevés de 10 à 60 mètres par siècle qui mettent en péril des habitations situées au lieu-dit l'Avancée. À cet endroit et sur environ $600 \mathrm{~m}$ de long, la falaise de marne et d'argile, instable, est soumise à des glissements importants. La dynamique de recul s'est accélérée ces dernières années en raison d'une pluviométrie particulièrement abondante depuis 1997 (Gress, 2000 ; Lahousse et Pierre, 2002).

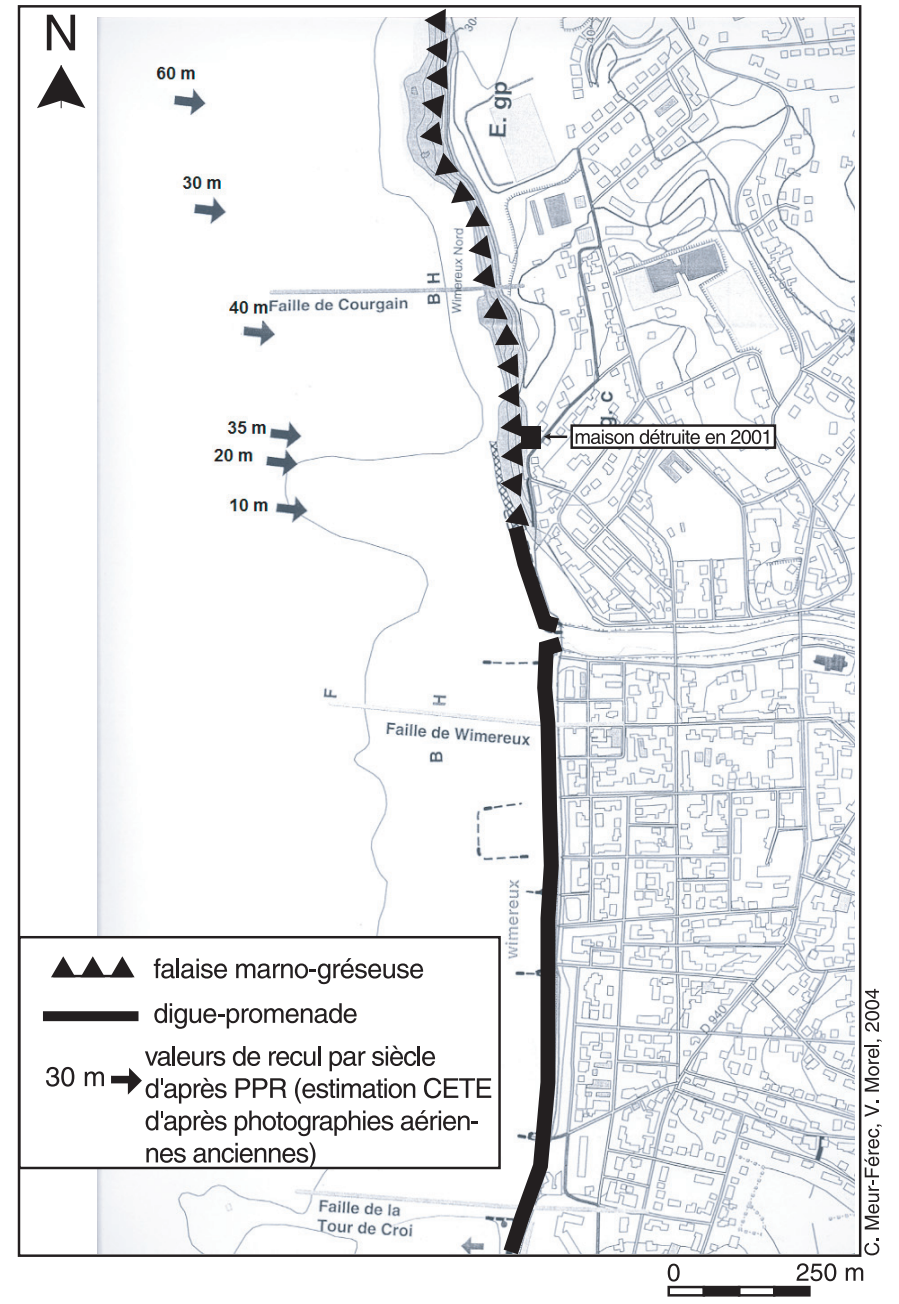

Fig. 2. Extrait modifié du projet du «PPR Falaises», 2002, réalisé par le Centre d'études techniques de l'Équipement du NordPas-de-Calais.

Ces données attestent non seulement de l'ancienneté de la tendance érosive caractérisant la dynamique côtière de Wimereux, mais également de la connaissance du phénomène par les services de l'État, la mairie et les propriétaires depuis au moins le dix-neuvième siècle.

De façon générale sur les littoraux urbanisés, malgré la propension au recul de nombreux secteurs côtiers, l'occupation humaine a eu tendance à effectuer une translation vers la mer.

\section{Glissement vers la mer et densification des installations humaines}

$\mathrm{Au}$ cours des dernières décennies, la frange côtière française est devenue un espace où se concentrent des enjeux sans cesse grandissants (Ifen, 1996; Meur-Férec, 2003b). On est passé progressivement du «territoire du vide » du dix-huitième siècle (Corbin, 1988) au « littoral d'empoigne » (Paskoff, 1998). 


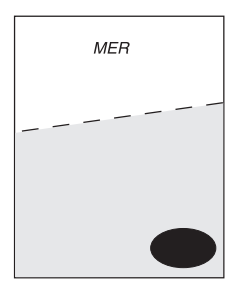

Jusqu'au XIXe s., villages en retrait de la côte.

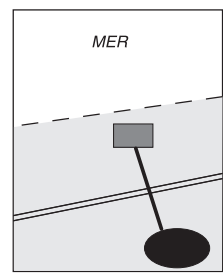

XIXe s., naissance des stations balnéaires.

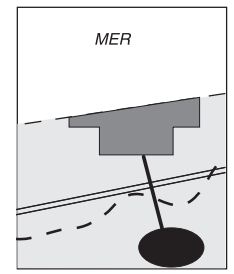
extension de l'urbanisation le long de la côte.
XXe s., essor des stations et

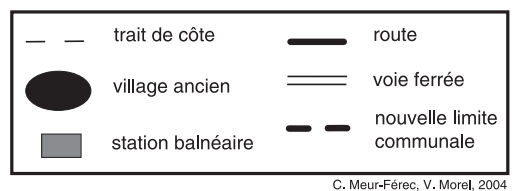

Fig. 3. Schéma du transfert du bâti vers la côte.

Certains chiffres, notamment ceux publiés par l'Ifen (2000), reflètent l'intensité et l'actualité du phénomène. Ainsi, les communes côtières qui ne couvrent que $4 \%$ du territoire métropolitain concentrent $10 \%$ de la population, $12 \%$ des logements neufs construits entre 1980 et 1996 et $37 \%$ des capacités d'hébergements touristiques. La densité de population dans ces communes (272 hab./ $\left./ \mathrm{km}^{2}\right)$ est deux fois et demie supérieure à la moyenne nationale et peut être multipliée par dix durant la saison estivale (Shirmann-Duclos et Laforge, 1999). Plus on est proche du rivage, plus ces problèmes augmentent. Ainsi, en moyenne, $15 \%$ de la frange côtière des 0-500 m étaient occupés par des espaces bâtis en 1960, contre 31 \% en 1990 (Robin et Verger, 1996).

L'essor du tourisme balnéaire au début du vingtième siècle, puis sa « démocratisation » (Deprest, 1997) sont les principaux facteurs de densification de l'occupation du littoral. La plupart des villages anciens qui se situent à quelques kilomètres du trait de côte se sont dédoublés, après la Seconde Guerre mondiale, par la création d'une station balnéaire implantée sur le rivage (Fig. 3).

Wimereux illustre ce phénomène qui apparaît à travers l'analyse des séries diachroniques de cartes et la littérature, révélant les étapes et les processus de territorialisation de cet espace côtier (Cochois, 1999; Klein, 1998; Latteux, 1999 ; Sintive, 1991) (Fig. 1).

Avant 1804 et l'installation par Bonaparte d'un immense camp militaire dans l'optique d'un débarquement en Angleterre, Wimereux est un site vierge. Après le départ de l'armée, un hameau de pêcheurs dépendant de la commune de Wimille se développe autour du port. Le contexte politique, économique et social de la seconde moitié du dix-neuvième siècle favorise ensuite l'émergence d'une station balnéaire. Ainsi, une ligne maritime régulière pour Folkestone, une route côtière (D 940) et une ligne de chemin de fer désenclavent le secteur au cours des années 1840 à 1870. Dans les années 1850 et 1860, des propriétaires privés achètent à l'État des terrains en front de mer et, en 1859, le premier hôtel ouvre.
Une opération immobilière d'envergure, entreprise par deux spéculateurs parisiens en 1867, amorce la construction de la station balnéaire. À la fin des années 1860, la trame de base et la délimitation du site par la voie ferrée conditionnent définitivement le développement de la station : les constructions et les activités ne cessent dès lors de se densifier sur la frange côtière. Dès les années 1880, la vocation balnéaire de Wimereux est nettement marquée et la station connaît une certaine réputation. Wimereux devient en 1899 une commune à part entière, indépendante de Wimille. La station est déclarée troisième station climatique maritime de France en 1913. Durant la Première Guerre mondiale, les hôtels sont réquisitionnés pour servir d'hôpitaux militaires, puis la clientèle de convalescents, en majorité anglaise, revient en villégiature dans les années vingt, donnant à la station son caractère britannique. En 1930, Wimereux est à la deuxième place des stations balnéaires de la région pour le nombre d'équipements, derrière le Touquet. Outre son orientation balnéaire, Wimereux devient aujourd'hui un secteur résidentiel attractif pour les actifs de Boulogne. Tournée vers la mer et valorisant la qualité de son environnement côtier et de son patrimoine historique, elle connaît un essor économique et démographique sans commune mesure avec sa "commune mère », Wimille, restée rurale et en retrait du développement balnéaire.

Parallèlement à l'haliotropisme touristique (Corlay, 1995), les côtes françaises connaissent aussi dans la seconde moitié du vingtième siècle une densification des activités liée à l'essor et à la modernisation des industries et des ports. Toutes ces mutations profondes d'un espace traditionnellement peu occupé augmentent considérablement les enjeux sur la frange côtière. Une telle concentration d'enjeux sur un espace à fortes contraintes environnementales, restreint et mobile, a pour conséquence une croissance importante de la vulnérabilité. Recul du trait de côte vers la terre, glissement des installations humaines vers la mer, cette dynamique convergente, à l'origine des risques côtiers, reflète une tendance générale observée sur la plupart des littoraux urbanisés (Fig. 4). En réponse à cet état de fait, la gestion des risques d'érosion côtière s'est progressivement développée.

\section{La gestion des risques liés à la mobilité du trait de côte}

En France, l'État est le principal acteur en matière de gestion des risques d'érosion côtière. Son rôle consiste à garantir une politique coordonnée sur le long terme alliant protection des lieux habités (travaux de défense contre la mer) et prévention (Bourrelier, 1997; Direction des Transports maritimes, des Ports et du Littoral (DTMPL), 2002; Dubois-Maury, 2001). De leur côté, 


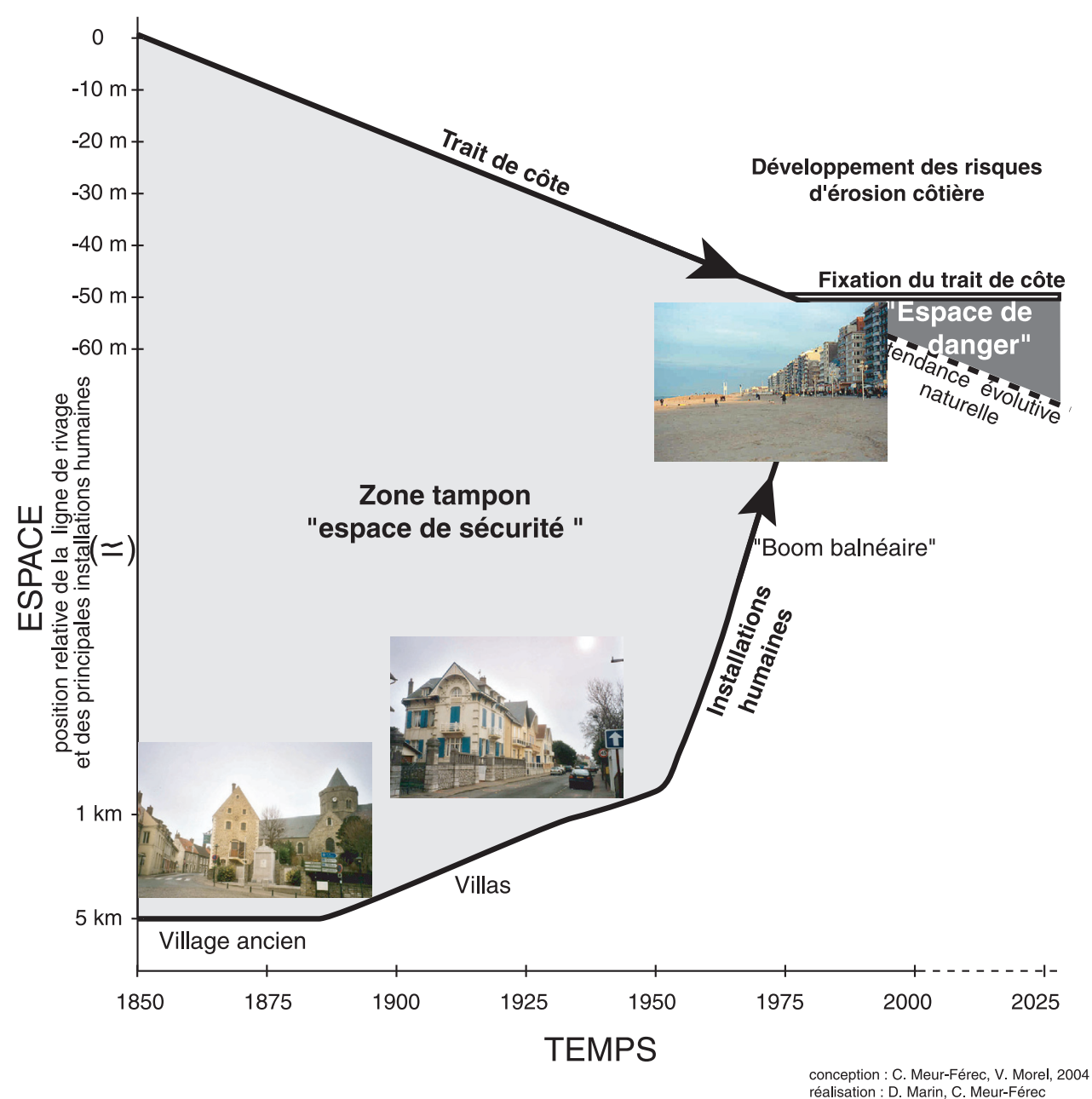

Fig. 4. Schéma de la convergence des dynamiques du trait de côte et de l'occupation du rivage : l'émergence des risques côtiers.

les élus locaux sont confrontés sur le terrain à de très lourdes responsabilités et doivent souvent faire face à de sérieux dilemmes.

\section{Protection contre la mer}

La construction d'ouvrages de défense efficaces contre la mer, comme les digues et les barrages, est techniquement possible, et les Néerlandais en donnent un exemple mondialement célèbre avec le Plan Delta (Miossec, 1998a). Le problème majeur n'est pas tant la maîtrise technique de ces ouvrages que les coûts qu'ils représentent et les choix de société qu'ils impliquent. En France, la loi de 1807, relative « au dessèchement des marais, aux travaux de navigation, aux routes, aux ponts, aux rues, places et quais dans les villes, aux digues et aux travaux de salubrité dans les communes " constitue le texte de référence en matière de défense contre la mer. Elle donne aux riverains la responsabilité de se protéger et à l'Etat la possibilité d'intervenir financièrement s'il l'estime "utile et juste », en cas d'intérêt public. «La priorité générale de l'État est de favoriser les opérations s'inscrivant dans une logique globale, faisant l'objet d'une évaluation coût-avantage » (DTMPL, 2002). Depuis $1999^{2}$, il est demandé aux préfets chargés de ces dossiers de privilégier les opérations exemplaires, quitte à majorer très largement le taux d'intervention et d'éviter ainsi tout saupoudrage. Les taux de subvention peuvent ainsi atteindre $80 \%$ d'aide publique cumulée. L'État consacre des sommes importantes et croissantes à la défense des lieux habités. Suite aux avenants apportés pour faire face aux dégâts engendrés par les tempêtes de 1999, le total atteint presque $60 \mathrm{M} €$ pour la période 2000 à 2006 (DTMPL, 2002).

Comme sur la plupart des côtes urbanisées, des problèmes de défense contre la mer apparaissent à Wimereux au fil du développement de l'occupation humaine. Dès la fin du dix-neuvième siècle, les propriétaires qui s'inquiètent pour leurs biens sollicitent l'aide de l'État. La requête faite en 1884 par les vingt-huit propriétaires est

\footnotetext{
${ }^{2}$ Le décret $\mathrm{n}^{\circ}$ 99-1060 du 16 décembre 1999 relatif aux subventions de l'État pour des projets d'investissement est entré en vigueur à compter du $1^{\mathrm{er}}$ avril 2000.
} 
refusée par les services de l'État qui estiment que : «[...] La seule dégradation de la côte est l'envahissement naturel de la mer qui, de temps immémoriaux, tend à ronger toute la côte comprise entre la pointe de la Crèche et le cap Gris-Nez [...]. Nous ne voyons dans l'intérêt engagé [...] que les intérêts privés des propriétaires qui voient diminuer leurs propriétés de jour en jour. Dans ces conditions, l'État n'entend pas contribuer de ses deniers à l'exécution des travaux de défense demandés. » Cette réponse correspond strictement à la logique de la loi de 1807. Dès lors, les particuliers et la commune s'organisent pour lutter contre l'érosion. Dès 1889, les projets de digues privées et les demandes d'autorisations de délimitation du domaine public maritime (DPM) aux services maritimes se multiplient. En 1902, un syndicat de propriétaires pour la défense du trait de côte se crée. À partir de 1911, des épis et des clayonnages sont construits sur la plage, financés par la commune qui contracte un emprunt remboursé par une imposition extraordinaire. La commune et le syndicat de propriétaires se lancent, entre 1922 à 1927, dans la construction des trois tronçons de la digue-promenade. Cette dernière, totalement détruite lors de la Seconde Guerre mondiale, est reconstruite entre 1947 et 1949 et payée au titre des dommages de guerre. Depuis, la dynamique hydrosédimentaire malmène les ouvrages. Lors des tempêtes de l'hiver 1967, une grande brèche éventre la digue; les travaux sont pris en charge à hauteur de $30 \%$ par le ministère de l'Équipement et de $30 \%$ par le conseil général. À nouveau, en 1971, 1982 et 1990, des travaux de réfection de la digue, partiellement subventionnés par l'État, doivent être entrepris après plusieurs tempêtes. L'accroissement et la concentration des enjeux sur la côte justifient probablement davantage aujourd'hui qu'au dix-neuvième siècle l'intervention de l'État au titre de l'intérêt public.

\section{Prévention}

Parallèlement aux mesures de protection contre la mer, l'État mène une politique de prévention des risques, visant à réduire la vulnérabilité, notamment par la maîtrise des enjeux (limitation des constructions dans les secteurs à forts aléas). Depuis la loi du 2 février 1995 relative «au renforcement de la protection de l'environnement ", les PPR sont désormais le principal outil de prévention des risques dits «naturels" (Encadrés 3 et 4). Le but des PPR est d'améliorer la sécurité des personnes, d'arrêter la croissance de la vulnérabilité des biens et des activités dans les zones exposées et, si possible, de la réduire afin de limiter les indemnisations qui sont largement supportées par la collectivité nationale (MATE, 1997b). La réglementation des PPR interdit d'aménager des terrains et de construire dans les zones d'aléas les plus forts (zones rouges). Cette réglementation est très stricte si la sécurité des personnes est en jeu. Prescrit par le préfet et réalisé par les services de l'État, le PPR est annexé aux plans locaux d'urbanisme (PLU), après concertation avec les élus locaux. Une fois approuvé, le PPR devient opposable aux demandes de permis de construire et autres autorisations d'occupation du sol régies par le code de l'urbanisme. La priorité générale de l'État est de favoriser les opérations s'inscrivant dans une logique globale, faisant l'objet d'une évaluation coût-avantage permettant de démontrer la pertinence des orientations. Le budget alloué à la prévention des risques naturels a connu une forte augmentation ces dernières années. En 2002, 15 M€ ont été consacrés aux PPR. L'État privilégie la prévention sur le rivage ainsi que sur les sites où le maître d'ouvrage se sera engagé à mettre en place un suivi du trait de côte (DTMPL, 2002).

Dans le Pas-de-Calais, le préfet a prescrit en 1998 un PPR concernant les risques d'érosion côtière qui se décline en deux volets : un «PPR Falaises » et un «PPR Zones basses ». Sa réalisation a été confiée au SMBC et au CETE. À Wimereux, outre les problèmes d'entretien de la digue-promenade et d'abaissement de l'estran concomitants, la principale préoccupation en matière d'érosion est aujourd'hui l'évolution de la falaise nord qui inquiète fortement les riverains et les collectivités locales. En effet, en arrière de cette falaise instable se développe une zone urbanisée fortement menacée par le recul. Ce secteur est inclus dans le «PPR Falaises » dont le projet a été soumis en 2001 à l'avis des élus municipaux (Fig. 2). Or le conseil municipal a émis à l'unanimité un avis défavorable sur ce projet, estimant que le recul y était sous-évalué. De ce fait, la «zone rouge » serait, soit trop étroite si on ne fait pas de travaux de protection de la falaise, soit trop large si on la protège. Selon leur propre estimation, s'appuyant sur une étude hydrogéotechnique commandée à un bureau d'études par la commune et cofinancée par les fonds européens Interreg II (Gress, 2000), les élus estiment qu'en l'absence d'ouvrage, plus de 100 maisons seraient menacées d'ici un siècle. De son côté, le CETE a calculé qu'au rythme actuel du recul, moins de 10 maisons seraient concernées à cette échéance. Ce désaccord retarde l'approbation du PPR et son application. Cependant, l'aval des élus locaux n'est pas nécessaire à l'approbation du PPR et le préfet peut décider sa validation après enquête publique.

Indirectement, la loi Littoral du 3 janvier 1986 « relative à l'aménagement, la protection et la mise en valeur du littoral » contribue aussi à diminuer la vulnérabilité en interdisant ou en limitant les constructions proches de la mer, notamment dans la bande côtière des 100 mètres et sur les «espaces proches du rivage ». Elle impose, de plus, la délimitation dans les PLU d'espaces naturels « remarquables » strictement protégés de toute urbanisation. Cependant, cette restriction ne concerne pas les zones déjà urbanisées, comme c'est le cas à Wimereux. 


\section{Encadré 3. Les risques dits « naturels»}

L'expression « risques naturels » est très largement utilisée, notamment au niveau institutionnel et réglementaire, lorsque l'on se réfère à des aléas considérés comme étant d'origine naturelle (séismes, tempêtes, glissements de terrain, etc.). Ce qualificatif «naturel » s'utilise notamment par opposition aux risques dits « technologiques ». Cependant, si certains aléas sont indubitablement d'origine naturelle (volcans ou séismes), le risque, qui résulte des enjeux et de la vulnérabilité des sociétés, ne peut être qualifié, à proprement parler, de naturel. De plus, de nombreux aléas qui apparaissent a priori naturels, comme les glissements de terrain ou les inondations, sont, dans les milieux fortement anthropisés, sensiblement influencés par les aménagements humains. Ainsi, il est communément admis que la mobilité du trait de côte peut être accentuée par des phénomènes anthropiques (Paskoff, 1993 ; Pinot, 1998). R. D'Ercole et P. Pigeon (2000) voient même dans l'appellation risques naturels « une lecture manichéiste et naïve du couple aléa et vulnérabilité (opposant nature d'une part et culture de l'autre), faisant implicitement référence à une sorte de paradis perdu, naturel ». Ils rappellent «l'évidente anthropisation des aléas dits naturels ».

\section{Encadré 4. L'érosion des espaces naturels : un risque qui n'en est pas un?}

Une ambiguïté subsiste souvent lorsque l'on parle de risque d'érosion sur des espaces naturels. À partir du postulat que le risque est soumis à l'existence d'enjeux, le recul du trait de côte dans un secteur préservé au titre des espaces naturels (propriété du CELRL, par exemple) ne constitue pas un risque en soi. A contrario, on pourrait soutenir que le milieu naturel lui-même et les espèces qu'il abrite constituent un enjeu à défendre contre l'érosion. Cependant, est-il possible d'affirmer que ce qui serait perdu, par une invasion marine par exemple, serait plus précieux que ce qui résulterait de ce changement : modification de la flore et de la faune, redynamisation du milieu, augmentation possible de la biodiversité? L'érosion elle-même, en tant que processus dynamique naturel, ainsi que les « paysages d'érosion » qu'elle génère (Mottier, 2003) pourraient avoir autant, voire plus, de valeur écologique et scientifique qu'une côte stabilisée. Ces considérations n'ont bien sûr de sens que s'il n'existe pas, en arrière de la côte, d'enjeux indirectement menacés, comme par exemple des zones inondables habitées ou exploitées, ou d'espèces en voie de disparition sur le site.

\section{L'option du retrait}

Outre les mesures de prévention, la loi du 2 février 1995 offre la possibilité de recourir à l'expropriation pour cause d'utilité publique des biens exposés à certains « risques naturels majeurs menaçant la vie humaine». Cette procédure concerne uniquement les risques "prévisibles » dans les cas où il existe une menace grave et où les coûts de sauvegarde et de protection de la population sont supérieurs aux indemnités d'expropriation et aux coûts de démolition des constructions menacées. Il s'agit d'une disposition restée jusqu'à présent exceptionnelle, lourde à appliquer (enquête publique et déclaration d'utilité publique) et relevant uniquement de l'État. Cependant, cette procédure vient d'être assouplie et décentralisée par la loi du 30 juillet 2003 relative «à la prévention des risques technologiques et naturels et à la réparation des dommages $»^{3}$. Cette dernière autorise désormais les communes et leurs groupements à prendre l'initiative et à être bénéficiaires de l'expropriation pour cause de risque naturel majeur mettant en péril la vie humaine. Il existe actuellement une quarantaine de dossiers de ce type en France, mais un seul concerne le littoral,

\footnotetext{
${ }^{3}$ Dans son article 60, cette loi modifie l'article L. 561-1 du code de l'environnement dans lequel la phrase : « les biens exposés à ce risque peuvent être expropriés par l'État » est remplacée par la phrase : «l'État peut déclarer d'utilité publique l'expropriation par lui-même, les communes ou leurs groupements, des biens exposés à ce risque ».
}

celui de la falaise de Criel-sur-Mer où une dizaine de maisons seront prochainement détruites.

À Wimereux, cette option n'a pas été clairement envisagée. Cependant, le désaccord entre services de l'État et municipalité sur le nombre de maisons menacées par le recul de la falaise est révélateur des difficultés d'application de ce texte. En effet, selon la loi de 1995, les coûts de protection doivent être comparés aux coûts d'indemnisation pour expropriation. Or, la méconnaissance du nombre de constructions concernées rend impossible ce calcul. D'après les estimations de la municipalité, qui souhaite la construction d'une digue pour arrêter le recul de la falaise et prolonger la promenade côtière, le coût de travaux de protection (estimé à $6 \mathrm{M} €$ dont $80 \%$ demandés en subventions à l'État et aux collectivités territoriales) serait nettement inférieur au coût d'expropriation (estimé à $38 \mathrm{M} €$ pour 170 maisons). Le rythme de recul futur étant inconnu de tous, le calcul prescrit par la loi de 1995 s'avère extrêmement difficile. Une analyse économique purement quantitative s'avère donc impossible et ne peut pas remplacer une prise de décision politique.

Bien que peu populaire, la possibilité d'expropriation et de retrait constitue une alternative lorsque les autres solutions sont proscrites. Davantage utilisée en Angleterre (MAFF, 1993), elle est probablement appelée à se développer en France. En effet, selon la direction des Transports maritimes, des Ports et du Littoral (2002), « dans certaines zones présentant un risque pour les personnes (falaises côtières par exemple), la procédure 
d'expropriation liée à la prévention des risques naturels doit impérativement être envisagée, voire privilégiée car, dans ces zones, aucuns travaux ne permettent d'apporter une garantie absolue de sécurité pour les habitants ». Cette option marque une nouvelle approche consistant à envisager le retrait des installations humaines, en phase cette fois avec la dynamique de recul du rivage. Elle entraîne la disparition du risque par la suppression des enjeux.

\section{Les responsabilités et les dilemmes des élus locaux}

Outre l'État, les communes ont de lourdes responsabilités en ce qui concerne les risques. En matière de police municipale, le maire est le premier responsable avant la mise en place des plans de secours par l'État. L'article L 2212 du code général des collectivités locales précise que la police municipale a pour objet «d'assurer le bon ordre, la sûreté, la sécurité et la salubrité publiques ». Cela implique notamment : « le soin de prévenir, par des précautions convenables, et de faire cesser, par la distribution des secours nécessaires, les accidents et les fléaux calamiteux ainsi que les pollutions de toute nature, tels que les incendies, les inondations, les ruptures de digues, les éboulements de terre ou de rochers, les avalanches ou autres accidents naturels, les maladies épidémiques ou contagieuses, les épizooties, de pourvoir d'urgence à toutes les mesures d'assistance et de secours et, s'il y a lieu, de provoquer l'intervention de l'administration supérieure ». Il incombe donc aux maires de prendre des arrêtés de péril et (ou) de démolition s'ils redoutent un risque imminent sur leur commune.

Le maire de Wimereux a dû recourir à cette procédure en ordonnant, le 7 novembre 2000, suite à l'avis des experts, l'évacuation d'une maison située au bord de la falaise nord. Une semaine plus tard, la terrasse s'effondra effectivement et le pignon de la maison se fissura. Le 18 mai 2001, exerçant à nouveau son pouvoir de police pour la sécurité des habitants, le maire prit un arrêté de démolition de la maison menacée. La mairie a ainsi dû avancer près de $100000 €$ pour l'étude d'impact, les travaux de démolition et le raccord des réseaux. Parallèlement, elle demanda un arrêté de catastrophe naturelle, conditionnant le remboursement par les assurances, qui est publié au Journal officiel du 29 mai 2001.

Aujourd'hui, le recul de la falaise se poursuit, mais le dossier reste en suspens. La mairie n'a pas obtenu les subventions pour les travaux prévus en 2001, car l'utilité publique ne semble pas clairement démontrée pour les services de l'État. Une dizaine de maisons, menacées à une échéance incertaine, justifient-elles de tels investissements publics? De leur côté, certains dénoncent l'immobilisme des pouvoirs publics et même de la mairie face à l'urgence de la situation. Ainsi, un article de la Voix $d u$ Nord titre le 24 janvier 2001 : «La falaise recule, que le dossier avance! » La possibilité d'expropriation n'est pour l'instant pas clairement évoquée. Dans ce contexte de très fort aléa et en l'absence de mesures de prévention, la vulnérabilité s'accroît...

Le difficile dossier de l'érosion de la falaise nord de Wimereux illustre la complexité des situations auxquelles sont confrontés les élus locaux. Leurs responsabilités, souvent perçues comme écrasantes, associées aux pressions exercées par les électeurs pour défendre leurs biens contre la mer les placent en situation délicate lorsqu'il s'agit de gérer des problèmes d'érosion côtière. La construction d'ouvrages de protection coûteux les tente naturellement puisque ces travaux réduisent l'aléa et la vulnérabilité, tout en préservant et même en accroissant les enjeux (la valeur d'un bien solidement protégé et bénéficiant de la proximité immédiate de la mer est évidemment majorée). La position des services de l'État, qui refusent souvent de subventionner des travaux pharaoniques au motif qu'ils ne répondent pas à l'intérêt général ou qu'ils dépassent en coût l'expropriation des riverains, est souvent mal perçue au niveau local.

\section{Conclusion}

Si l'exemple de Wimereux reste encore un cas particulier en France, il est cependant caractéristique des problèmes qui se poseront dans les prochaines décennies sur de nombreux littoraux urbanisés.

Animées par des dynamiques convergentes, l'évolution naturelle et l'occupation humaine du rivage ont abouti au cours du siècle passé à un prévisible « télescopage ». Il en découle l'émergence, puis la multiplication des risques d'érosion côtière.

En raison des fortes contraintes environnementales auxquelles les rivages sont soumis, l'occupation de la frange côtière entraîne inévitablement des surcoûts de protection contre la mer et d'entretien des ouvrages. Or, au regard des aménités apportées par la proximité immédiate de la plage, et des revenus qui en sont tirés, ces contraintes ont souvent été ignorées ou minimisées. La situation actuelle est le résultat d'une évolution spontanée qui s'est faite le plus souvent sur des critères d'intérêts particuliers, sans vision à long terme, ni choix clair en matière de politique publique. Dans l'optique d'un développement durable et face aux difficultés croissantes que pose le problème de l'érosion côtière, la nécessite de faire des choix apparaît aujourd'hui. Plusieurs textes réglementaires marquent l'essor des préoccupations dans ce domaine, mais un des problèmes majeurs est qu'en règle générale, la vision de l'État diffère sensiblement de la position des élus locaux. Ces divergences de point de vue ralentissent, voire bloquent la mise en place des mesures de gestion. Or, il y a urgence, pas tant pour empêcher des habitations de s'effondrer sur la plage, accidents encore 
isolés, mais pour réfléchir à une stratégie à long terme (protéger ou se retirer, selon les cas). Arrêter l'essor des enjeux dans les zones de forts aléas permettrait de limiter la croissance des coûts supportés par la collectivité.

\section{Remerciements}

Nous adressons nos remerciements au personnel de la mairie de Wimereux ainsi qu'à celui du Service maritime des ports de Boulogne-sur-Mer et Calais pour nous avoir ouvert leurs archives. Nous tenons également à remercier les relecteurs de la revue pour leurs suggestions et leurs commentaires fructueux.

\section{Références}

Battiau-Queney, Y., Billet, J.-F., Chaverot, S., Lanoy-Ratel, P., 2003. Recent shoreline mobility and geomorphologic evolution of macrotidal sandy beaches in the north of France, Marine Geology, 194, 31-45.

Bird, E.C.F., 1987. Human activity and environmental processes, Wiley and sons Ltd.

Bourrelier, P.H., Comité interministeriel de l'évaluation des politiques publiques, Commissariat Général au Plan, 1997. La Prévention des risques naturels, rapport d'évaluation, Paris, La Documentation française.

Cochois, C., 1999. Mémoire en images de Wimereux, Joué-lès-Tour, A. Sutton.

Corbin, A., 1988. Le Territoire du vide, l'Occident et le désir de rivage 1750-1840, Paris, Flammarion.

Corlay, J.-P., 1995. Géographie sociale, géographie du littoral, Norois, 42, 165, 247-265.

Costa, S., Delahaye, D., Freire-Diaz, D., Davidson, R., Laignel, B., Di Nocera, L., 2002. Quantification par analyse photogrammétrique du recul des falaises et des apports en galets corrélatifs, Actes du symposium international From experts opinion to modelling, attribute to Pr Jean-Claude Flageollet, European Center of Geomorphological Hazards, 205-214.

Crowell, M., Douglas, B.C., Leatherman, S.P., 1997. On forecasting future US shoreline positions: an test of algoritms, Journal of Coastal Research, 13, 4, 1245-125.

Dauvin, J.-C. (Ed.), 2002. Gestion intégrée des zones côtières : outils et perspectives par la préservation du patrimoine naturel, Muséum national d'histoire naturelle.

Deprest, F., 1997. Enquête sur le tourisme de masse, Paris, Belin.

D’Ercole, R., 1994. Les vulnérabilités des sociétés et des espaces urbains, concepts, typologie, mode d'analyse, Revue de géographie alpine, $\mathrm{n}^{\circ}$ spécial 4, 87-96.

D'Ercole, R., Pigeon, P., 1999. L'expertise internationale des risques dits naturels : intérêt géographique, Annales de géographie, 608, 339-357.

D'Ercole, R., Pigeon, P., 2000. L'évaluation du risque à l'échelle internationale. Méthodologie et application aux diagnostiques préalables aux actions de préparation et de prévention des catastrophes, Cahiers savoisiens de géographie, 1, 2936.

DTMPL (Direction des Transports maritimes, des Ports et du Littoral), 2002. Quel rôle pour l'État en matière de défense contre la mer. Note disponible sur le site internet du ministère de l'Équipement.
Dubois-Maury, J., 2001. Les Risques naturels, quelles réponses? Paris, La Documentation française.

Gaillot, S., Chaverot, S., 2001. Méthode d'étude des littoraux à faible évolution. Cas du delta du Golo (Corse) et du littoral du Touquet (Pas-de-Calais) en France, Géomorphologie : relief, processus, environnement, 1, 47-54.

Gress, A., 2000. Instabilité du secteur rue E. Zier. Rapport du bureau d'études hydrogéotechnique pour la mairie de Wimereux.

Hénaff, A., Lageat, Y., Costa, S., Plessis, E., 2002. Mobilités du recul des falaises du Pays de Caux. Actes du symposium international From experts opinion to modelling, attribute to Pr Jean-Claude Flageollet, European Center of Geomorphological Hazards, 235-242.

Ifen, 1996. Pressions humaines sur le littoral. Des pressions qui se diversifient sur un espace toujours vulnérable, Les Données de l'environnement, 19.

Ifen, 2000. La pression de la construction ne se relâche pas sur le littoral métropolitain, Les données de l'environnement, 55.

Klein, R., 1998. La Côte d'Opale, architecture des années 20 et 30, Institut français d'architecture, 75-98.

Lageiste, J., 2002. Le tourisme : facteur de territorialisation du détroit du Pas-de-Calais?, Hommes et terres du Nord, 2, 21-25.

Lahousse, P., Pierre, G., 2002. Le recul des falaises crayeuses du Blanc-Nez (Pas-de-Calais, France). Actes du symposium international "From experts opinion to modelling ", attribute to Pr Jean-Claude Flageollet, European Center of Geomorphological Hazards, 235-244.

Latteux, N., 1999. Wimereux d'hier et d'aujourd'hui, Paris, le Livre d'histoire.

MAFF (Ministry of Agriculture, Food and Fisheries), 1993. Coastal defence and the environment. A guide to good practice. Environment Matters.

Marquet, A., 2000. Étude sur la variabilité spatio-temporelle du trait de côte et sur les conditions météo-marines en baie de Wissant. Mémoire de maîtrise de géographie, Université du Littoral Côte d'Opale.

MATE (Ministère de l'Aménagement du territoire et de l'Environnement), Ministère de l'Équipement, des Transports et du Logement, 1997a. Plans de prévention des risques littoraux (PPR), Paris, La Documentation française.

MATE (Ministère de l'Aménagement du territoire et de l'Environnement), Ministère de l'Équipement, des Transports et du Logement, 1997b. Plans de prévention des risques naturels prévisibles (PPR), guide général, Paris, La Documentation française.

Meur-Férec, C. (Ed.), 2003a. La Vulnérabilité des territoires côtiers : évaluation, enjeux et politiques publiques. Rapport final, Contrat IFREMER 2002/1140 717.

Meur-Férec, C., 2003b. L'occupation du littoral, ou le choix d'investir un territoire à risques, Océanis, 28, 1/2, 115-137.

Miossec, A., 1998a. Les sociétés face à l'élévation contemporaine du niveau de la mer, Annales de géographie, 600, 201-219.

Miossec, A., 1998b. Les littoraux, entre nature et aménagement. SEDES.

Moore, L.J., 2000. Shoreline mapping technics. Journal of Coastal Research, 16-1, 111-124.

Morel, V., 2002. Les hommes et les falaises : des rapports d'intérêts différents dans l'espace. Actes du colloque Les falaises de Picardie, état des lieux, enjeux, actions, avril 2001, Amiens, 109-116.

Mottier, A., 2003. Le Contact entre terre et mer à Wissant. Mémoire de diplôme de $5^{\mathrm{e}}$ année de l'École nationale supérieure de la nature et des paysages, Blois. 
Paskoff, R., 1993. Côtes en danger, Paris, Masson.

Paskoff, R., 1998. Les Littoraux, impact des aménagements sur leur évolution, Paris, Masson.

Paskoff, R., 2001. L'Élévation du niveau de la mer et les espaces côtiers, le mythe et la réalité, Paris, Institut océanographique.

Paxion, K., Cohen, O., 2002. La baie de Wissant : cent ans d'évolution en images, Mappemonde, 67/3, 24-27.

Pigeon, P., 2002. Réflexions sur les notions et les méthodes en géographie des risques dits naturels, Annales de géographie, 627-628, 452-470.

Pinot, J.-P., 1998. La Gestion du littoral. Littoraux tempérés, côtes rocheuses et sableuses. Paris, Institut océanographique.

Reçu le $1^{\mathrm{er}}$ septembre 2003. Accepté le $1^{\mathrm{er}}$ avril 2004.
Pirazzoli, P., 1993. Les Littoraux, Paris, Nathan.

Robin, M., 2002. Étude des risques côtiers sous l'angle de la géomatique, Annales de géographie, 627-628, 471-501.

Robin, M., Verger, F., 1996. Pendant la protection, l'urbanisation continue, Les Ateliers du Conservatoire du littoral, 13.

Sabatier, F. et Suanez, S., 2003. Evolution of the Rhone Delta coast since the end of the 19th century, Géomorphologie: Relief, Processus, Environnement, 4, 283-300.

Shirmann-Duclos, D., Laforge, F., 1999, La France et la mer, PUF. Sintive, E., 1991, Étude du patrimoine de Wimereux (Pas-de-Calais), Lille, DIREN Nord-Pas-de-Calais.

To access this journal online: www.edpsciences.org 